\title{
Advanced Impedance-based Control Design for Decoupling Multi-Vendor Converter HVDC Grids
}

\author{
Adedotun J. Agbemuko, Graduate Student Member, IEEE, José Luis Domínguez-García, Member, IEEE, \\ Eduardo Prieto-Araujo, Member, IEEE, and Oriol Gomis-Bellmunt, Senior Member, IEEE
}

\begin{abstract}
A progressive interconnection of existing HVDC links to form grids and the development of completely new HVDC grids from different vendors are expected shortly. One of the current challenges of such endeavour is unintended interactions due to independently designed controllers. This article proposes a design methodology for decentralized controllers to mitigate such interactions in multi-vendor voltage source converter (VSC)HVDC grids. The approach presented relies on the unique standalone input-output impedance transfer function of each VSC, and the global impedance transfer function as seen from each terminal after interconnection with other VSCs. Subsequently, network-level controllers are designed by attempting to match the global responses at selected locations based on a novel interaction analysis, to the unique transfer function model of the vendors at the corresponding location. This approach reduces the entire problem to an impedance matching problem. We demonstrate the efficacy and flexibility of both the methodology and the designed controllers in mitigating interactions due to the independent design of VSC controllers through nonlinear simulations on a four-terminal droop controlled HVDC grid.
\end{abstract}

Index Terms-VSC-HVDC, DC-DC interactions, multi-vendor HVDC grid, impedance-based analysis, $\mathcal{H}_{\infty}$ control

\section{INTRODUCTION}

$\mathbf{O}$ $\mathrm{NE}$ of the major advantages of voltage source converter high voltage direct current (VSC-HVDC) transmission is the potential for multi-terminal DC (MTDC) grids. Literature suggests this will be the network architecture for HVDC grids in the future [1]. As of today, almost all VSC-HVDC projects implemented are HVDC links and nearly all are based on single vendor designs, where vendors often apply in-house solutions based on varying experiences and proprietary design strategies. In the near future, it is expected that these links from several vendors will be progressively interconnected to share resources, responsibilities, and overall improvement of efficiency [2]-[4]. Additionally, it is expected that newly built

Adedotun J. Agbemuko and José Luis Domínguez-García are with the Electrical Power Systems Area, Institut de Rrecerca en Energia de Catalunya (IREC), 08930, Barcelona, Spain (e-mails: \{aagbemuko,jldominguez\}@irec.cat).

Eduardo Prieto-Araujo and Oriol Gomis-Bellmunt are with the Electrical Engineering Department, Centre d'Innovaciò Tecnològica en Convertidors Estàtics i Accionaments (CITCEA-UPC), Universitat Politècnica de Catalunya, Barcelona 08028, Spain (e-mails:\{eduardo.prieto-araujo, oriol.gomis\}@upc.edu).

This work was financially supported by the European Union's Horizon 2020 research and innovation programme under Marie-Sklodowska-Curie action INCITE - "Innovative controls for renewable source integration into smart energy systems", grant agreement No. 675318 .

This work has also been partially funded by FEDER/Ministerio de Ciencia, Innovación y Universidades - Agencia Estatal de Investigación, Project RTI2018-095429-B-I00 and by the ICREA Academia program. networks would be multi-vendor converter based. Therefore, it will be desired that multi-vendor systems can be seamlessly interconnected without issues.

The HVDC community is generally aware of the emerging issues of multi-vendor systems and one of the most important is the interoperability of such independently designed systems. An industry-led European Union (EU) funded project delved deeply into the issue of interoperability in all aspects by identifying potential sources of challenges including control architectures and dynamics [5]. However, little is known on formal approaches to solving some identified challenges. Interoperability issues usually result from intellectual property protection leading to the impossibility of sharing explicit information. Even when there is some shared information, converter designs could be so different that efforts at integrating these converters result in a tedious and time-consuming process. Moreover, if retuning of converter controllers is required, vendors may be averse to such changes without a clear framework due to potential dependencies of several control loops. Besides, such retuning may never guarantee the robustness of a multivendor system [6].

Numerous researchers have made several proposals to improve the interoperability and interaction analysis of multivendor meshed HVDC grids [7]-[9]. However, proposals mainly rely on state-space approaches where explicit knowledge of all states is implied [7], [8]. It is well known that state-space methods do not support independent control design, thus, unavoidable interaction results [10]. In [5] authors proposed to adapt local control parameters based on real-time digital simulations of the interconnection of converter replicas from several vendors. Authors went further in implementing dedicated controllers to limit adverse interactions [2]. However, a methodology for adaptation and control design was not provided.

Despite the growing efforts to seamlessly integrate converters from multi-vendors, vendors are often held to standards such that at in stand-alone, a converter is designed to behave optimally and predictably across expected operating range [11]. Therefore, the local behaviour pre-connection to a grid is often acceptable and vendors may be willing to provide masked transfer function responses of such behaviours. On the alternative, such transfer responses can be estimated from black-box models provided by the vendors. An advantage of transfer functions is that they mask the underlying structure of a converter and the corresponding control systems and architecture. Therefore, this pre-connection guarantee can be exploited as a reference model to design dedicated network- 
wide decoupling controllers.

In this paper, we contribute towards the realization of multivendor systems by proposing a methodology for decentralized control design of network-level controllers to mitigate interactions due to differences in control dynamics. To design the said controllers, the equivalent impedance model of each VSC is analytically derived. Then, droop gains that meet a bounded frequency requirement are selected for each VSC to guarantee an acceptable response before interconnection with an HVDC grid. Subsequently, the VSCs are interconnected through the HVDC grid to obtain the equivalent global transfer functions and the system is partitioned into two components from each controllable node. Then, interaction analysis is carried out and controllers are designed to decouple network-level interactions by manipulating the tractable components of the partitioned system from each identified controllable node. To demonstrate the efficacy and flexibility of the methodology and designed controllers in mitigating interactions, nonlinear simulations are carried out on a four-terminal droop controlled VSC-HVDC grid.

\section{SiMPLIFIEd IMPEDANCE-BASED MODELLING OF SUBSYSTEMS}

For the sake of brevity since the focus of this article is on the DC side, a simplified, yet accurate modelling procedure is adopted. The detailed modelling procedures can be found in [12], [13]. The components on the AC side that impact the behaviour on the DC side - inner-loop, AC filter, PLL (phaselocked loop), are integrated through the inner-loop referenceto-output transfer function.

It is important to make clear at this juncture that the main interest in this section is to obtain the final input-output impedance response of each subsystem, although analytically. This is due to the lack of availability of actual vendor models of VSCs. Hence, the use of established generic control architecture to demonstrate the methodology for advance control design. However, if actual black or grey-box models are available from a vendor, system identification methods can be adopted to obtain the final impedance response. Therefore, the knowledge of the control architecture and parameters although shown are not necessary. A realistic system identification work is postposed to a future article.

\section{A. Impedance Models of Droop/Active Power Controlled VSCs}

Fig. 1 illustrates a generic single-line diagram of a VSC terminal and control-block diagram. The control system is implemented in the synchronous reference frame (SRF) - $d q$ frame, facilitated by the PLL. The final goal is to obtain the equivalent input-output impedance, representative of the VSC as a transfer function in stand-alone. This can be obtained by deriving the closed-loop response for a given control strategy employed at the local level.

For a converter in droop/active power control mode, both modes can be combined in an analytical modelling procedure such that the value of droop gain determines the actual control mode. That is, constant power control mode can be obtained by setting the droop gain $R_{d c}=0$. Fig. 2 shows the

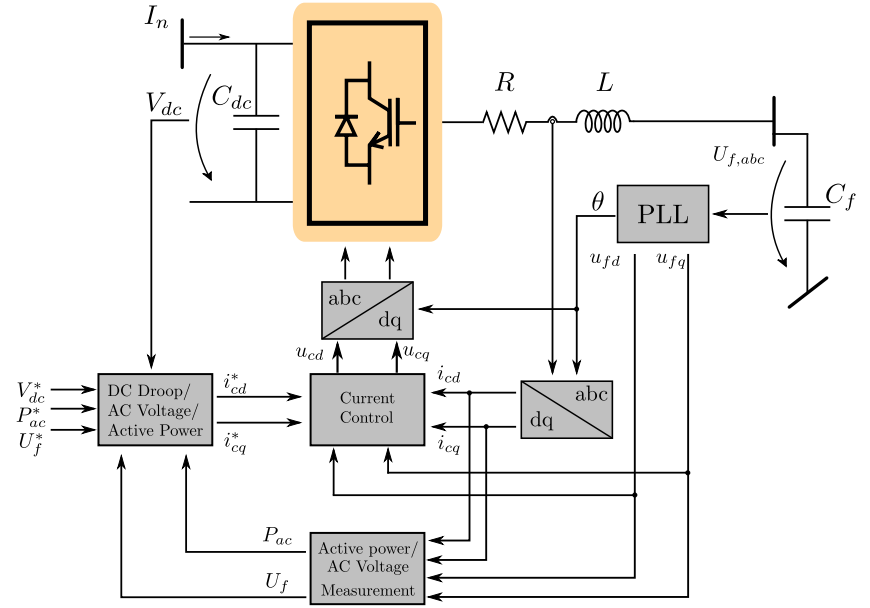

Fig. 1. Single-line terminal and control-block layout of a generic VSC

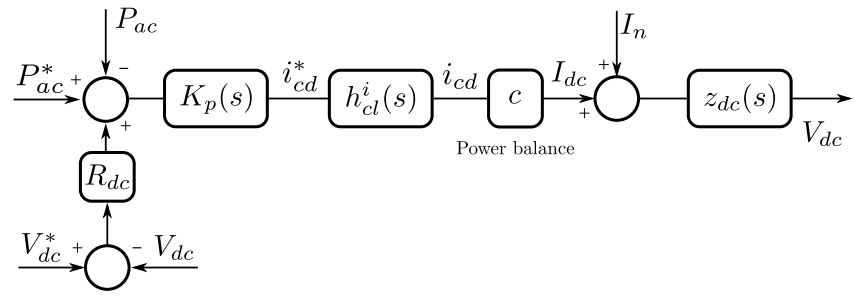

Fig. 2. Simplified control-block diagram of a droop/active power VSC

simplified closed-loop block diagram of an active power/droop controlled VSC. The closed-loop response of the $i^{t h}$ converter in droop/active power mode can be obtained as

$$
V_{d c, i}=H_{c l, i}^{v_{d p}}(s) V_{d c, i}^{*}+H_{c l, i}^{d p}(s) P_{a c, i}^{*}+z_{o c, i}(s) I_{n, i}
$$

where $V_{d c, i}^{*}$ is the scheduled voltage reference, $V_{d c, i}$ is the terminal DC voltage, $P_{a c, i}^{*}$ is the scheduled power reference; whereas, $H_{c l, i}^{v_{d p}}$ is the closed-loop reference to output transfer function (this cancels out in active power mode when $\left.R_{d c, i}=0\right), H_{c l, i}^{d p}(s)$ is the power reference to DC voltage closed-loop transfer function; $I_{n, i}$ is the total DC-bus current flowing through the VSC, and $z_{o c, i}(s)$ is the equivalent inputoutput impedance. At an operating point, (1) can be linearized as

$$
\Delta V_{d c, i}=z_{o c, i}(s) \Delta I_{n, i}
$$

where $z_{o c, i}(s)$ can be obtained from Fig. 2 as

$$
\begin{aligned}
z_{o c, i}(s) & =\frac{z_{d c, i}(s)}{1+H_{o l, i}^{v_{d p}}(s)} \\
& =\frac{z_{d c, i}(s)}{1+z_{d c, i}(s)\left(\frac{h_{c l}^{i}(s) K_{p}(s) u_{f d i}^{0}\left(I_{d c, i}^{0}+R_{d c, i}\right)}{k V_{d c, i}+h_{c l}^{i}(s) K_{p}(s) u_{f d, i}^{0} V_{d c, i}^{0}}\right)}
\end{aligned}
$$

where $z_{d c, i}(s)$ is the primitive impedance of the equivalent DC capacitance, $K_{p}(s)$ is the active power PI compensator, $h_{c l}^{i}(s)$ is the closed-loop gain of the inner-loop, $H_{o l, i}^{v_{d p}}$ is the openloop gain from DC voltage reference to output; whereas, $I_{d c, i}^{0}$, $V_{d c, i}^{0}, u_{f d, i}^{0}$ are the operating points of converter DC injection current, DC and AC (at point-of-common coupling) voltages respectively, and $k=2 / 3$ is the power variant constant. 


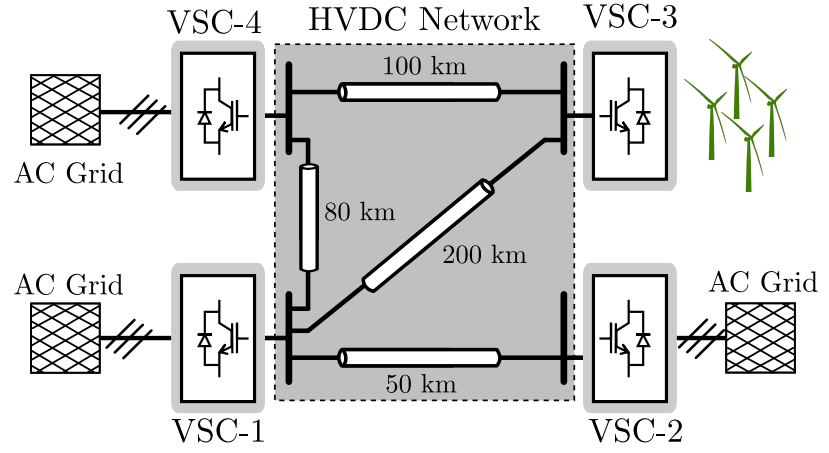

Fig. 3. Single-line diagram of a four-terminal HVDC grid with independently designed VSCs

\section{B. Selection of Droop Gains and Converter-level Response}

To provide context, in this article a four cable HVDC grid is to be interconnected with four independently designed VSCs. It is worth to remark that a more realistic system may arise from the expansion of existing HVDC links for which two converters are concurrently designed by the same vendor. Fig. 3 shows the interconnection of the VSC-HVDC grid. The grid operator identified three terminals to provide droop response as required. Therefore, VSCs-1, 2, and 4 are equipped with droop control, whereas VSC-3 is connected to an offshore intermittent power source in constant power control mode. The active power loops of the converters at VSCs-1, 2, and 4 are designed with first-order responses of $15 \mathrm{~ms}$, $4 \mathrm{~ms}$, and $30 \mathrm{~ms}$ respectively. These time responses are arbitrarily chosen to reflect potential diversity of responses and may differ for different HVDC implementations. However, typical values for real implementations are typically around five to fifteen times slower than the inner-loop [14], [15]. The inner-loop is assumed standardized across all terminals with a time response of $1 \mathrm{~ms}$.

The impacts of droop gains are most visible at the global level where more than one converter may be equipped to provide droop response. Hence, the appropriate droop gains in stand-alone that meet specific global requirements (such as a power-sharing formula) may not be known ahead of time during the independent design phases of each converter. To fit this into the described methodology, droop gains may be selected during independent design to meet a bounded requirement in the frequency domain. This is only to guarantee an acceptable behaviour pre-connection for which it is desired that the global response (after interconnection to an arbitrary grid) will mimic after supplementary control design if desired. For a maximum allowed voltage deviation of $\Delta V_{d}^{\max }$ p.u. from rated voltage, and maximum expected bus current change of $\Delta I$ p.u. of rated direct current of each converter, the bounded requirement in the frequency domain can be computed from (2) according to [16]

$$
z_{o c, i}^{\max }=20 \log _{10}\left(\frac{\Delta V_{d, i}^{\max } \times V_{\text {rat }}}{\Delta I_{i} \times I_{\text {rat }, i}}\right) \mathrm{dB}
$$

where $z_{o c, i}^{\max }$ is the maximum allowed magnitude of impedance response imposed by droop control, $\Delta V_{d c, i}^{\max }$ is the maximum allowed voltage deviation in p.u. at the $i^{t h}$ VSC, $V_{\text {rat }}$ is the

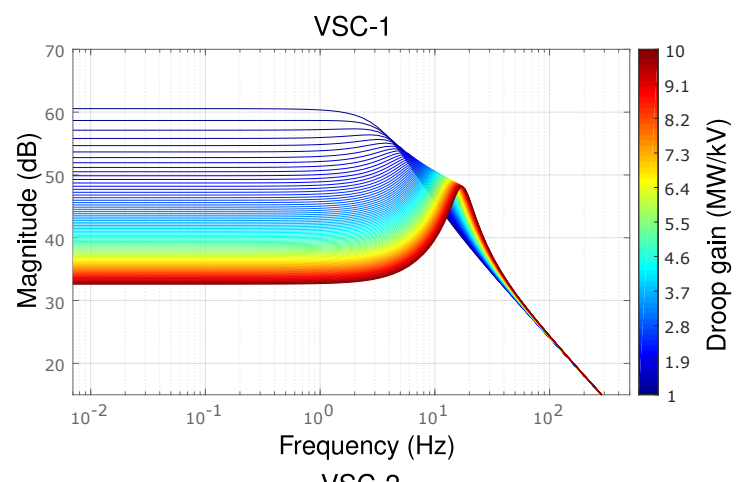

VSC-2

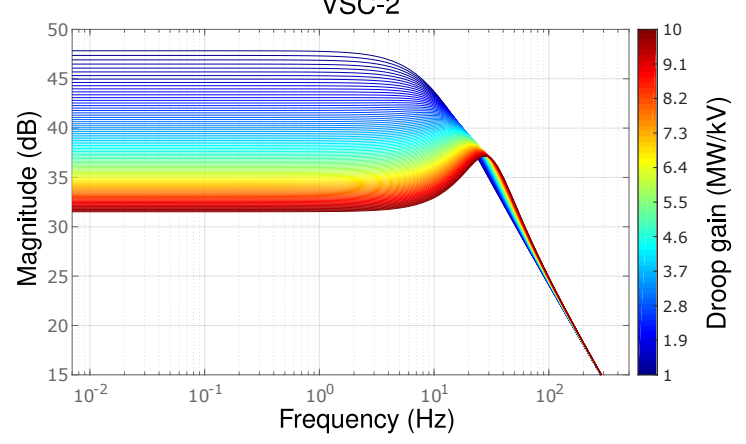

VSC-4

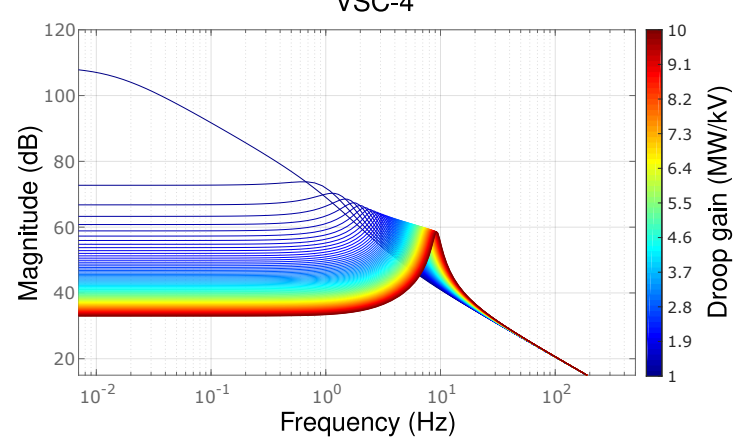

Fig. 4. Vendor specific terminal impedance response at an operating point for varying droop gains

rated DC voltage of the grid, $\Delta I_{i}$ is the maximum expected DC bus current change in p.u., $I_{r a t, i}$ is the rated current of the $i^{t h}$ VSC.

To determine the required droop gain at each converter that meets a bounded frequency requirement, Fig. 4 shows the frequency responses of the equivalent input-output impedance of each VSC (before interconnection) showing the impacts of variation of droop response from $1-10 \mathrm{MW} / \mathrm{kV}$. In general, for all terminals, the increasing droop response reduces the steady-state gain below $1 \mathrm{~Hz}$ where droop response is most active. Additionally, for VSC-1 and VSC-4 where the active power response is orders slower than VSC-2, increasing the droop gain also isolates low-frequency resonances that may be aggravated after interconnection despite a reduction of the steady-state gain. Therefore, the droop gain should not be unnecessarily high to prevent resonances, or too low to avoid breaching steady-state limits.

For a maximum allowed voltage deviation of 0.15 p.u. and the maximum expected change in DC-bus current of $\Delta I=0.25$ p.u. at each VSC (equivalent to the contribution of $25 \%$ of rated power to droop response), and other required 


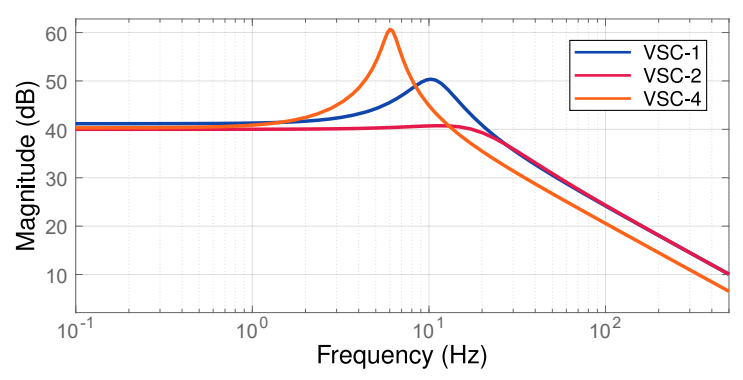

Fig. 5. Unique impedance response of each VSC corresponding to selected droop gains

parameters in Table III, a bound of $\approx 42 \mathrm{~dB}$ can be established and droop gains that meet this bound are selected. Due to the inherent differences in the first-order responses of each VSC, the droop gains that meet the bounded frequency response will differ as well. Hence, VSC-2 is expected to have the lowest droop gain and negligible oscillatory response for the same bound compared to VSC-1 and VSC-4; whereas, VSC-4 is expected to have the highest gain and the most oscillatory response. It is important to note that a system operator may set different physical bounds on each converter in the global system relative to the priority assigned to each terminal. Additionally, actual deviation margins will be lower after interconnection than those obtained at the local level, depending on network resistances, and the interaction with other droop gains. The approximate droop gains of each terminal equipped with droop control that meets the defined bound for VSC-1, 2, and 4 are 4.12, 3.37, and $4.86 \mathrm{MW} / \mathrm{kV}$ respectively.

To verify that the selected droop gains satisfy the requirements pre-connection, Fig. 5 shows the impedance response of each terminal corresponding to the selected droop gain. These are the equivalent DC impedance responses of each VSC that may be provided by a vendor or estimated from input-output responses. Importantly, these responses mask all related information about a VSC, its internal structure, control structure, among other things. Fig. 6 shows the nonlinear timedomain responses to a step response of $200 \mathrm{MW}(\approx 25 \%$ of rating) at the terminals of each vendor. As can be observed, all steady-state deviations meet the bounded requirement of a maximum 1.15 p.u. of DC voltage. Furthermore, the observed resonances match the expected behaviour as seen from Fig. 5 ; that is the impedance responses can be estimated from input-output time responses. Specifically, VSC-4 has the most oscillatory behaviour; whereas, VSC-2 is the least oscillatory. The oscillatory behaviour is not necessarily of concern at the local level as actual oscillation frequencies may shift after interconnection, and the role of the global level controller is to mitigate such.

In summary, these responses are in general acceptable. However, local oscillatory behaviour as seen from frequency responses may encourage the vendor of a converter to retune locally before interconnection. It will be highlighted in the following how the local frequency responses are completely reshaped after interconnection, showing the real challenge for DC network operators.

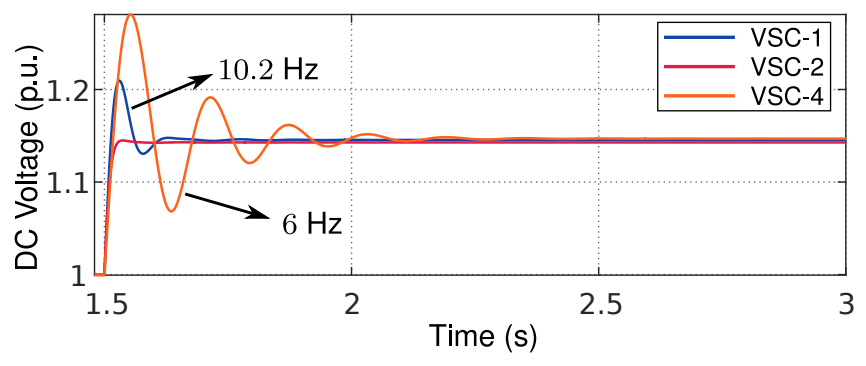

Fig. 6. Converter specific nonlinear time response of local behaviour to terminal changes

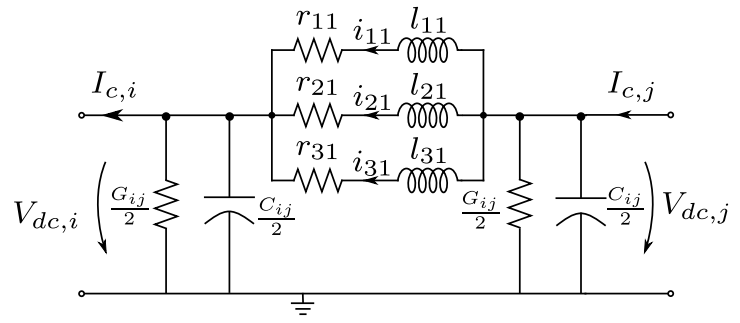

Fig. 7. Single-section distributed $\pi$ cable model

\section{DC Cable Impedance Model}

A single $\pi$ distributed-section cable model has been utilized in this work to model the DC cable as shown in Fig. 7 with parameters given in Table I [17]. This choice is dependent on the frequency spectrum of interest which in this work is up to the bandwidth of the inner-loop at $\approx 150 \mathrm{~Hz}$ for which a single $\pi$ is sufficient [8]. Although more $\pi$ sections can be utilized to improve accuracy, the increased order only overwhelms the total order of the system without adding any relevant information. From Fig. 7, the equations of the cable model are given as

$$
\begin{aligned}
\frac{d i_{m 1}}{d t} & =\frac{V_{d c, j}}{l_{m 1}}-\frac{V_{d c, i}}{l_{m 1}}-\frac{i_{m 1}}{l_{m 1}} \quad(\forall m=1,2,3) \\
\frac{d V_{d c, j}}{d t} & =\frac{2 I_{c, j}}{C_{i j}}-\frac{2 I_{j, i}}{C_{i j}}-\frac{V_{d c, j} G_{i j}}{2 C_{i j}} \\
\frac{d V_{d c, i}}{d t} & =\frac{2 I_{j, i}}{C_{i j}}-\frac{2 I_{c, i}}{C_{i j}}-\frac{V_{d c, i} G_{i j}}{2 C_{i j}} \\
I_{j, i} & =\sum_{m=1}^{3} i_{m 1} ; \quad I_{n, i}=\sum_{i=1}^{n_{c}} I_{c, i}
\end{aligned}
$$

where $m$ is the number of branches in a section, $V_{d c, i}$ and $V_{d c, j}$ are the nodal voltages at the receiving and sending ends respectively, $I_{c, i}$ and $I_{c, j}$ are the currents injected into the corresponding bus from each connected cable; whereas $C_{i j}$ and $G_{i j}$ are the cable pole capacitance and admittance respectively, and $n_{c}$ is the total number of cables connected any bus as shown in Fig. 1. Subsequently, the equivalent impedance of the cable can be obtained by transforming the state-space representation into input-output impedance transfer function.

\section{INTERCONNECTION OF VSCS}

Following the modelling procedures in Section II, all subsystems are represented as equivalent impedance transfer func- 
TABLE I

DC CABLE PARAMETERS

\begin{tabular}{cccc}
\hline Variable & Value & Variable & Value \\
\hline$r_{11}$ & $0.1265 \Omega / \mathrm{km}$ & $l_{11}$ & $0.2644 \mathrm{mH} / \mathrm{km}$ \\
$r_{21}$ & $0.1504 \Omega / \mathrm{km}$ & $l_{21}$ & $7.2865 \mathrm{mH} / \mathrm{km}$ \\
$r_{31}$ & $0.0178 \Omega / \mathrm{km}$ & $l_{31}$ & $3.6198 \mathrm{mH} / \mathrm{km}$ \\
$C_{i j}$ & $0.1615 \mu \mathrm{F} / \mathrm{km}$ & $G_{i j}$ & $0.1015 \mu \mathrm{S} / \mathrm{km}$ \\
\hline
\end{tabular}

tions. Since impedance is a fundamental physical property of a network, the equivalent transfer functions can be interconnected as the physical structure of the system dictates.

\section{A. Global Response of the Interconnected System}

1) SISO Characterization of Network Responses: Due to interconnection, the expression of (2) must be modified to include the rest of the system. The modified voltage response at each terminal after interconnection can be obtained from the network impedance aggregation [13], [18] and expressed in vectorized form as

$$
\underbrace{\left(\begin{array}{c}
\Delta V_{1} \\
\Delta V_{2} \\
\Delta V_{3} \\
\Delta V_{4}
\end{array}\right)}_{\Delta \mathbf{V}}=\underbrace{\left(\begin{array}{llll}
z_{11}(s) & z_{12}(s) & z_{13}(s) & z_{14}(s) \\
z_{12}(s) & z_{22}(s) & z_{23}(s) & z_{24}(s) \\
z_{13}(s) & z_{23}(s) & z_{33}(s) & z_{34}(s) \\
z_{14}(s) & z_{24}(s) & z_{34}(s) & z_{44}(s)
\end{array}\right)}_{\mathbf{Z}_{c l}(s)} \underbrace{\left(\begin{array}{c}
\Delta I_{n, 1} \\
\Delta I_{n, 2} \\
\Delta I_{n, 3} \\
\Delta I_{n, 4}
\end{array}\right)}_{\Delta \mathbf{I}}
$$

where $\Delta \mathbf{V}$ is the vector of terminal voltage changes considering interconnection, $\Delta \mathbf{I}$ is the vector of potential bus currents changes, and $\mathbf{Z}_{c l}(s)$ is the global closed-loop impedance matrix. The matrix holds the information about the smallsignal dynamics, input-output stability, converter-converter interactions, and disturbance behaviour of the entire global system as a single-entity. The diagonal elements show the contribution of the rest of the system as seen at each terminal, whereas the off-diagonal elements in combination give an indication of system-wide interaction behaviour. Fig. 8 shows the SISO frequency responses of $\mathbf{Z}_{c l}(s)$ for each selected droop gains from previous section and the equivalent impedance response of each cable. The solid line indicates the calculated impedances and the star dots indicates the simulated impedances from nonlinear simulations. It can be observed the simulated results gives a good match with the derived impedances and an indication of accuracy. In addition, it is seen how the responses have been modified taking into account the impact of interconnection. Specifically, the SISO responses identify potential resonance frequencies in the system. A well damped mode around $10 \mathrm{~Hz}$ can be seen, a slightly damped mode around $33 \mathrm{~Hz}$, and poorly damped modes at $44.2 \mathrm{~Hz}$ and $73 \mathrm{~Hz}$; these are all interaction frequencies.

2) Relative Gain Array for Interaction Detection: Despite the resonant frequencies identified by the SISO responses, it is not obvious how each VSC is interacting with others. Thus, it becomes challenging to improve the global response as desired. Furthermore, it does not provide information on how
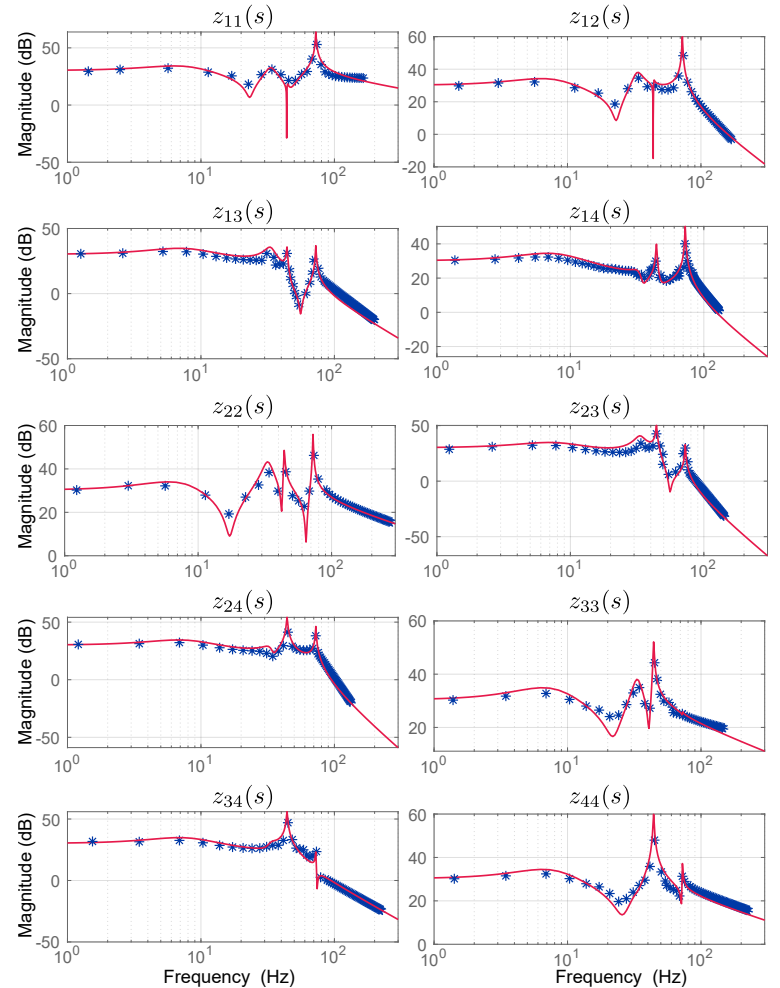

Fig. 8. SISO Frequency response of interconnected system: Analytical (red solid), simulation (blue star)

global controllers should be designed with inherent coordination. The frequency-dependent Relative Gain Array (RGA) is a tool that can assist in control design to mitigate system-level multi-input multi-output (MIMO) interactions [19]-[21]. The frequency-dependent RGA provides insights into how system inputs and outputs influence each other at each frequency through relative gains. In this case, the system consists of the disturbance behaviour modelled by $\mathbf{Z}_{c l}(s)$. The frequencydependent RGA can be computed as

$$
\begin{aligned}
\Lambda(\omega) & =\left(\begin{array}{cccc}
\lambda_{11}(\omega) & \lambda_{12}(\omega) & \cdots & \lambda_{1 n}(\omega) \\
\lambda_{21}(\omega) & \lambda_{22}(\omega) & \cdots & \lambda_{2 n}(\omega) \\
\vdots & \ldots & \ddots & \cdots \\
\lambda_{1 n}(\omega) & \lambda_{2 n}(\omega) & \cdots & \lambda_{n n}(\omega)
\end{array}\right) \\
& =\mathbf{Z}_{c l}(j \omega) \otimes\left(\mathbf{Z}_{c l}(j \omega)\right)^{-T}
\end{aligned}
$$

where $\otimes$ is the element-wise product (Hadamard product), $n$ is the number of subsystems, $\lambda_{i i}$ predicts the influence of changes at subsystem $i$ on itself and $\lambda_{i j}$ predicts the influence of changes at subsystem $j$ on $i$. That is, in a perfectly decoupled system, $\Lambda(\omega)$ is an identity matrix $\mathbf{I}$ at each frequency of interest. Hence, there is no relative amplification at a terminal for disturbances to itself and there is no transfer of dynamics between terminals since their coupling gain is 0 . Although a perfectly decoupled system may not be achievable in practice or necessary for interaction-free response, the RGA at desired frequencies should be as close to a unit matrix as possible.

For the global closed-loop impedance matrix $\mathbf{Z}_{c l}$, Fig. 9 shows the frequency-dependent RGA magnitude plot of $\mathbf{Z}_{c l}(s)$ 


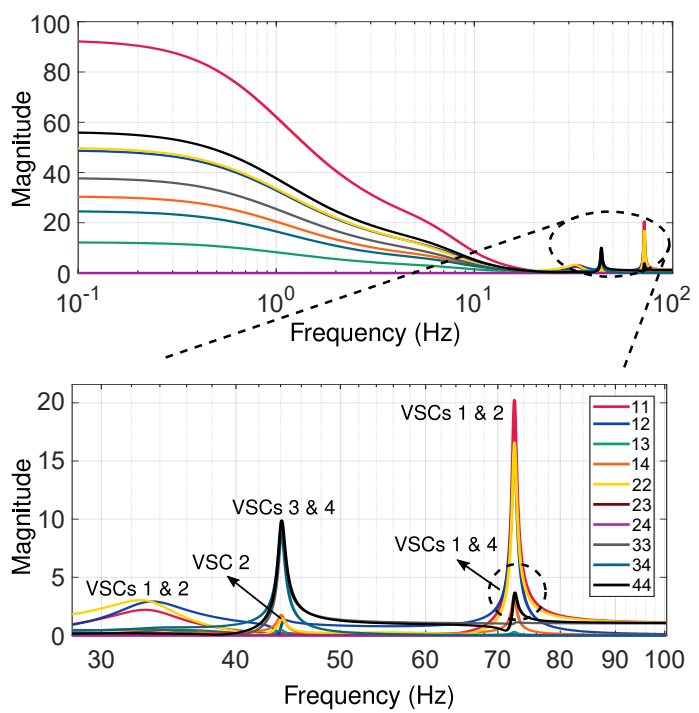

Fig. 9. Frequency-dependent relative gain array for interaction detection

showing the interactions between terminals, and their contributions to each identified interaction frequency. Interaction at a frequency is indicated by peaks in corresponding diagonal and off-diagonal elements. In this case, all terminals are strongly interacting in steady-state with VSC-1 expected to have the highest steady-state deviation margin followed by VSC-4, VSC-2, and VSC-3 respectively (identified by elements 11, $44,22,33)$. This is an expected effect of droop control.

In the oscillatory region; at the first fairly damped $33 \mathrm{~Hz}$ resonance, VSC-1 and VSC-2 are interacting at that frequency. At $44.2 \mathrm{~Hz}, \mathrm{VSC}-3$ and VSC-4 (indicated by elements 33, 44, $34)$ are the main contributors to that frequency with VSC2 contributing slightly. At $73 \mathrm{~Hz}, \mathrm{VSC}-1$ and VSC-2 are significantly interacting at this frequency. Thus, VSC-1 is the highest contributor to oscillatory behaviour in the system, and small disturbances at that terminal may aggravate the overall response. However, in this work, constant disturbances are mainly expected from VSC-3 where an intermittent power source is connected. Assuming this, the main oscillation frequency in the system will be around $44.2 \mathrm{~Hz}$. To summarize, the RGA plot indicates the best way to coordinate designed supplementary controllers by pairing up terminals and how many are indeed required.

Fig. 10 shows the DC voltage responses for a step disturbance at VSC-3. Immediately, it is clear how responses differ from those of Fig. 6. It can be observed that the oscillation frequencies match with one or more of those identified in Fig. 8. As predicted by the RGA, for disturbances at VSC-3, the dominant frequency is $44.2 \mathrm{~Hz}$. This is the case particularly for VSC-2, 3, and VSC-4 where $44.2 \mathrm{~Hz}$ is dominant with a lower magnitude at VSC-2. At VSC-1 the oscillatory responses are much lower as it is not contributing to this resonance. However, responses are more distorted as a result of its contribution to multiple frequencies in the system. Still, the dominant mode remains at $44.2 \mathrm{~Hz}$.

To further demonstrate the efficacy of the RGA plots in indicating how terminals are interacting, Fig. 11 shows the

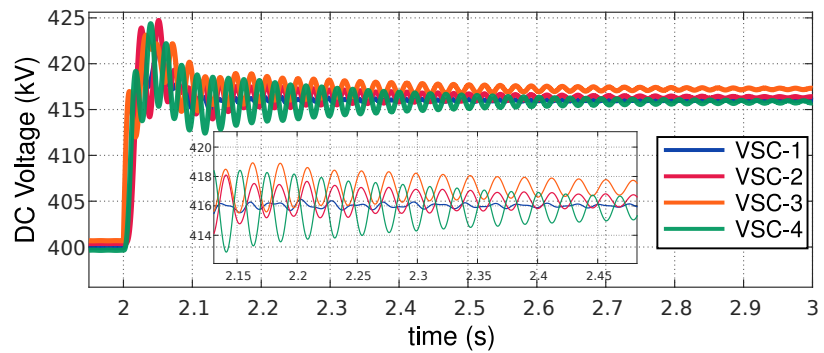

Fig. 10. DC voltage responses for a step disturbance at terminal VSC-3

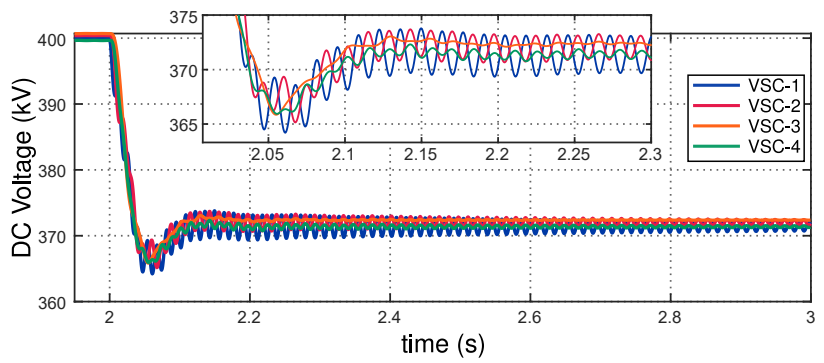

Fig. 11. DC voltage responses for a step disturbance at VSC-1

time-domain responses for step disturbances at VSC-1 instead. As expected, the dominant frequency predicted from the RGA plots analysis at VSC-1 is $73 \mathrm{~Hz}$ with VSC-2 interacting strongly with VSC-1. This can be observed from the voltage responses as the dominant frequency is $73 \mathrm{~Hz}$. Also, it shows that VSC-1, 2 are the major contributors, with VSC-4 only slightly at this frequency as indicated by the RGA plots; whereas VSC-3 has an acceptable response as it does not contribute to this frequency.

\section{B. Decomposition of MIMO to N SISO Problems}

Given the $\mathbf{Z}_{c l}(s)$ matrix, it is quite challenging to determine how to re-optimize the local controllers. However, it is clear from equation (6) that it is desired that $\Delta \mathbf{V}$ is uniformly as low and flat as control and constraints allow for guaranteed robust performance. Thus, the goal is to force the global responses of $\mathbf{Z}_{c l}(s)$ to mimic the unique local responses of each converter in Fig. 5 or few converters contributing most to interaction as shown in Fig. 9. Decomposing the global MIMO system into equivalent SISO responses for decentralized control involves partitioning the $\mathbf{Z}_{c l}(s)$ matrix into two components from each terminal-the local response based on (2) and transferred response from the rest of the network $z_{n e t, i}(s)$ as seen from the $i^{\text {th }}$ terminal. That is,

$$
z_{n e t, i}(s) \neq z_{n e t, j}(s)
$$

where $j$ is any other terminal and $z_{n e t, i}$ can be easily obtained by looking at a point-to-point link and generalizing to $N$ arbitrary terminals. For a point-to-point HVDC link with two terminals $-i, j$, the diagonal elements of $\mathbf{Z}_{c l}(s)$

$$
z_{i i}(s)=\frac{z_{o c, i}(s) z_{n e t, i}(s)}{z_{o c, i}(s)+z_{n e t, i}(s)}
$$

where $z_{n e t, i}(s)=z_{o c, j}(s)+z_{\text {cable }}(s)$. Clearly, the tractable component for control is $z_{n e t, i}$ and the non-tractable com- 
ponent is $z_{o c, i}$ which is the model of each vendor VSC. Therefore, at any terminal $i$

$$
z_{n e t, i}=\frac{z_{i i}(s) z_{o c, i}(s)}{z_{o c, i}(s)-z_{i i}(s)}
$$

$\Delta V_{i}$ at each terminal in the global system can be reformulated as [13]

$$
\Delta V_{i}=z_{o c, i}(s) \frac{1}{1+\frac{z_{o c, i}(s)}{z_{n e t, i}(s)}} \Delta I_{n, i} .
$$

Since $z_{o c, i}(s)$ is acceptable (and being a non-tractable component), then it may be desired that $z_{\text {net }, i} \rightarrow \infty$ as seen by terminal $i$. If this strictly holds, (11) simply reverts to (2) which is agreed as acceptable. Hence, in the interconnected system, each converter behaves as if it is in stand-alone if $z_{i i}(s) \approx z_{o c, i}(s)$. As can be seen, the entire problem reduces to an impedance matching problem and supplementary controllers are adopted to shape $z_{i i}(s)$ into $z_{o c, i}(s)$ or an approximation using the sensitivity of controllers to be obtained such that

$$
S_{i i}(s) z_{i i}(s)=z_{o c, i}(s) \quad \Longrightarrow S_{i i}(s) \approx \frac{z_{o c, i}(s)}{z_{i i}(s)}
$$

where $S_{i i}(s)$ is the target sensitivity transfer function of the controllers to be obtained. If only $m$ supplementary controllers are required, only the corresponding diagonal elements are required from the $\mathbf{Z}_{c l}(s)$ matrix.

\section{Controller Design Methodology}

The main objective is to reshape the corresponding $z_{i i}(s)$ into $z_{o c, i}(s)$ using the sensitivity of controllers to be determined. The main difference between the impedance response of each converter and the corresponding global responses is in the dynamic region where peaks can be seen. Therefore, the problem is to find a controller that flattens the peaks of $z_{i i}(s)$, subject to the established bounds, and other constraints such as controller order. This directly fits into the $\mathcal{H}_{\infty}$ decentralized fixed-structure framework [22].

\section{A. $\mathcal{H}_{\infty}$ Mixed-sensitivity Framework}

Fig. 12 shows the defined problem in the mixed-sensitivity framework where all matrices involved are fully diagonal. The generalized input to output expression of the formulation before supplementary design can be derived from Fig. 12 (dashed red box) as [19]

$$
\begin{gathered}
\left(\begin{array}{c}
\mathbf{z} \\
\Delta \mathbf{V}
\end{array}\right)=\underbrace{\left(\begin{array}{c|c}
0 & \mathbf{W}_{\Delta I_{s}} \\
\mathbf{W}_{\Delta v} \mathbf{Z}_{\text {diag }} & \mathbf{W}_{\Delta v} \\
\hline \mathbf{Z}_{\text {diag }} & \mathbf{Z}_{p}
\end{array}\right)}_{\mathbf{P}}\left(\begin{array}{c}
\Delta \mathbf{I} \\
\Delta \mathbf{I}_{s}
\end{array}\right) ; \mathbf{z}=\left(\begin{array}{c}
z_{1} \\
z_{2}
\end{array}\right) \\
\begin{array}{c}
\mathbf{Z}_{\text {diag }}(s)=\operatorname{diag}\left(z_{i i}, z_{j j}, \cdots, z_{m m}\right) \\
\mathbf{Z}_{p}=\operatorname{diag}\left(\alpha_{i i}, \alpha_{j j}, \cdots, \alpha_{m m}\right)
\end{array}
\end{gathered}
$$

where $\mathbf{W}_{\Delta v}$ is the system output weighting matrix, $\mathbf{W}_{\Delta I_{s}}$ is the control output weighting matrix, $\mathbf{z}$ is the weighted output to be reshaped, $\Delta \mathbf{I}$ is the input (bus current changes), $\Delta \mathbf{I}_{s}$ is the supplementary controller outputs, and $\Delta \mathbf{V}$ is the measured output. Whereas, $m$ is the total number of required supplementary controllers, $\mathbf{Z}_{\text {diag }}(s)$ consist of the corresponding diagonal elements of $\mathbf{Z}_{c l}(s)$ at nominal power flow and as many random power flows, $\mathbf{Z}_{p}$ is the 'fictitious plant' model consisting of scalar gains $\alpha$ corresponding to each terminal. The value of each $\alpha$ relative to others indicates the relative priority of each terminal. To include the controller(s) to be determined in the optimization framework, $\Delta \mathbf{I}_{s}=-\mathbf{K}_{s} \Delta \mathbf{V}$ is eliminated from (13) to obtain

$$
\begin{aligned}
& \mathbf{z}=\mathbf{N}(K) \Delta \mathbf{I} ; \quad \mathbf{N}(K)=\left(\begin{array}{c}
\mathbf{W}_{\Delta I_{s}} \mathbf{K}_{s} \mathbf{S Z}_{\text {diag }} \\
\mathbf{W}_{\Delta v} \mathbf{S Z}_{\text {diag }}
\end{array}\right) \\
& \mathbf{S}=\operatorname{diag}\left(S_{i i}, S_{j j}, \cdots, S_{m m}\right)
\end{aligned}
$$

where $\mathbf{N}(K)$ is the complete closed-loop formulation, $\mathbf{K}_{s}$ is a diagonal matrix of supplementary controllers with predefined structures and order (in this case a maximum order of three), $\mathbf{S}$ is the sensitivity matrix of supplementary controllers, and $\mathbf{S} \mathbf{Z}_{\text {diag }}$ is the reshaped global impedance response as described in (12). The optimization problem is to minimize $\mathbf{N}(K)$ such that

$$
\min _{K}\|\mathbf{N}(K)\|_{\infty} \leq \gamma
$$

where $\gamma=1$ is the maximum allowed peak in frequency domain [19].

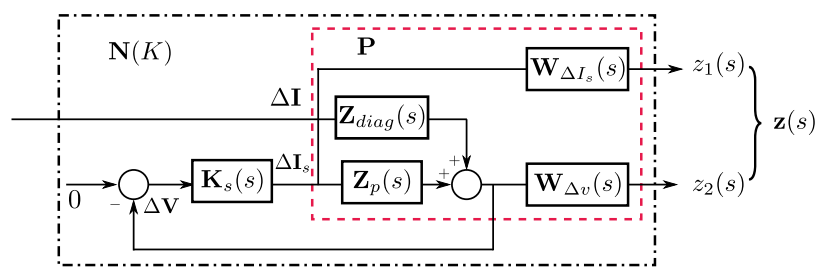

Fig. 12. Decentralized supplementary control design framework [19]

\section{B. Design Preliminaries}

1) Scaling: To maintain flexibility and a benchmark to assess performance, the bounds established in previous section ( $\Delta V_{d, \max }=0.15$ p.u. and $\Delta I=0.25$ p.u.) directly determine the limits within which supplementary controllers can act. In addition to these boundaries, maximum allowed control effort for each supplementary controller to match global impedance response to local response must be determined. In this paper, the maximum control effort defined in terms of current are $\Delta I_{s 1}^{\max }=0.3, \Delta I_{s 2}^{\max }=0.3, \Delta I_{s 4}^{\max }=0.2 \mathrm{p} . \mathrm{u}$., for each supplementary control respectively at the corresponding location. Each of these parameters could be chosen to reflect the desired response from each terminal distinctly. The described parameters $\left(V_{d, \max }, \Delta I\right.$, and $\left.\Delta I_{s}\right)$ are adopted to scale the system. Since the diagonal element corresponding to each terminal is sufficient to model the disturbance behaviour at that terminal with respect to the rest of the network, each terminal model is scaled according to

$$
\begin{aligned}
& z_{i i}^{s}(s)=\Delta V_{d, \text { max }}^{-1} z_{i i}(s) \Delta I \\
& z_{p}^{s}(s)=\Delta V_{d, \text { max }}^{-1} z_{p}(s) \Delta I_{s}
\end{aligned}
$$




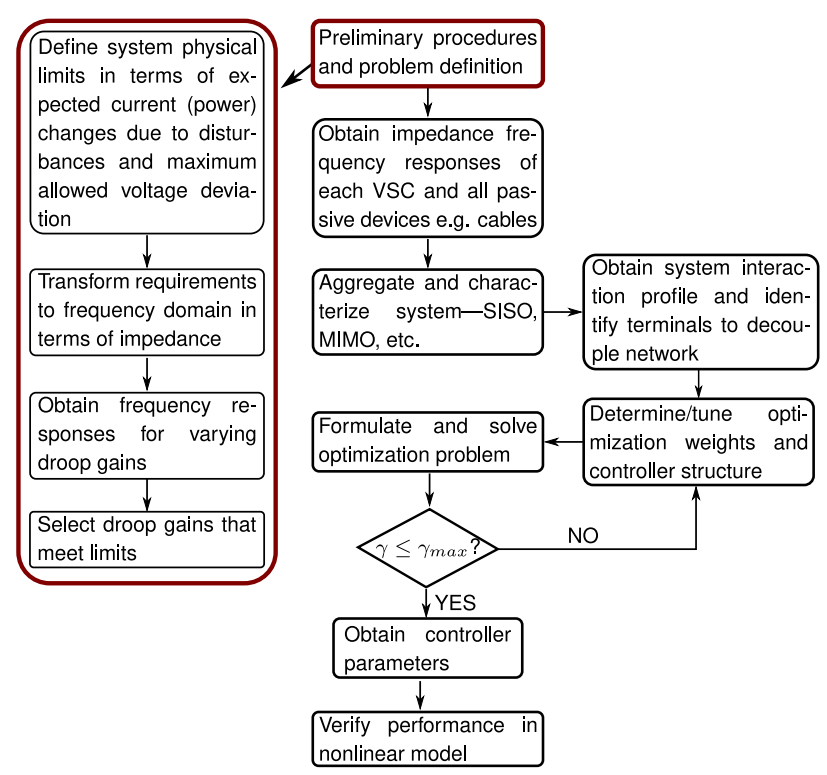

Fig. 13. Control design flowchart for droop controlled VSC-HVDC

where $z_{i i}^{s}(s)$ is the scaled disturbance model, and $z_{p}^{s}(s)$ is the scaled plant. Scaling ensures that the global impedance response is scaled to 1 p.u. in time and frequency domain.

2) Weighting Functions: The most important phase of the control design through $\mathcal{H}_{\infty}$ is the selection of weighting matrices, $\mathbf{W}_{\Delta v}(s)$ and $\mathbf{W}_{\Delta I_{s}}(s)$. Standard weights can be selected for $\mathbf{W}_{\Delta I_{s}}(s)$ if the maximum control bandwidth is known. This is important to prevent interactions, especially with the inner-loop [5]. The weights can be selected as a highpass filter with bandwidth equal to the maximum allowed, or the expected bandwidth of disturbances. In this case, the highest frequency of disturbance is around $70 \mathrm{~Hz}$. On the other hand, selection of $\mathbf{W}_{\Delta v}(s)$ is significantly simplified through equation (12) since $z_{i i}$ and $z_{o c, i}$ are known. Then, the output weighting function of each controllable terminal can be obtained based on an approximation of the target sensitivity function $S_{i i}(s)$ according to (17) with room for flexibility in the order of weights relative to desired complexity.

$$
w_{\Delta_{v i}(s)} \approx \frac{1}{S_{i i}(s)}
$$

where $S_{i i}(s)$ is previously defined in (12). The weighting functions for each controller to be determined are given in Table II.

3) Model Reduction: This is a prerequisite to a successful simple design and scalability to large DC grids. In this article, the Hankel norm is adopted to reduce the global responses at each location [19]. The model at VSC-1 was reduced from eighteen orders to six; whereas for each of VSC-2 and 4, the models were reduced from eighteen to eight.

Fig. 13 depicts an overview flowchart of the proposed methodology for advanced controller design as detailed in previous sections.

\section{Discussion and CAse Studies}

Case studies are performed on the four-terminal HVDC grid shown in Fig. 3 to establish the performance of several com-
TABLE II

WEIGHTING TRANSFER FUNCTIONS

\begin{tabular}{cccc}
\hline Location & $w_{\Delta_{v}}$ & \multicolumn{2}{c}{$w_{\Delta I_{s}}$} \\
\hline 1 & $\frac{1131 s}{s^{2}+1.885 s+0.03553}$ & $\frac{0.5 s}{s+2 \pi f_{1}} ;$ & $f_{1}=70 \mathrm{~Hz}$ \\
2 & $\frac{741 s}{s^{2}+1.662 s+0.02763}$ & $\frac{0.5 s}{s+2 \pi f_{2}} ;$ & $f_{2}=70 \mathrm{~Hz}$ \\
4 & $\frac{944.5 s}{s^{2}+1.885 s+0.03553}$ & $\frac{0.5 s}{s+2 \pi f_{4}} ;$ & $f_{4}=70 \mathrm{~Hz}$ \\
\hline
\end{tabular}

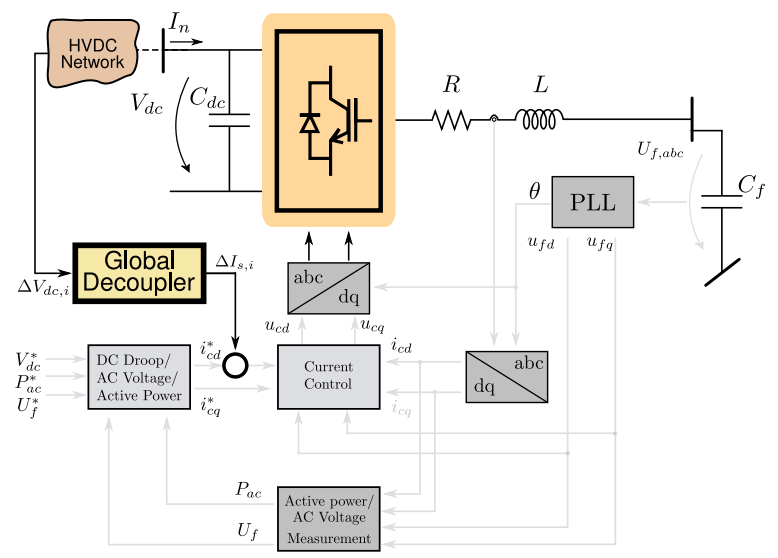

Fig. 14. Block diagram of synthesized global controllers relative to existing structure at any arbitrary terminal

binations of supplementary controllers at different locations, and dynamic performance to unexpected changes. The main goal is to establish any improvements in interaction induced responses with and without supplementary control.

Three dedicated decentralized supplementary controllers were designed for the system as seen from VSC-1, 2, and 4 with the flexibility to activate or deactivate any. VSC3 is assumed to integrate an offshore resource and deemed unsuitable for network-level supplementary control. Fig. 14 shows an overview of how the global control structure fits in with the existing local control. As long as an additional control channel is available, each designed global controller can be implemented in a dedicated control board external to the existing local control as suggested in [2]. On the alternative, each vendor may directly include the designed controllers as additional control boards on top of the local control. However, the operator is given sole access and responsibility of tuning and parametrizing the global controller as required within agreed constraints.

The frequency response of each decentralized supplementary controller at the desired locations is shown in Fig. 15. The controllers are designed by solving the $\mathcal{H}_{\infty}$ problem described by (15) subject to constraints previously highlighted. The realized gains for each synthesized controllers are $\gamma_{11}=0.472$, $\gamma_{22}=0.209$, and $\gamma_{44}=0.618$ at VSC-1, VCS-2, and VSC4 respectively. Since $\gamma<1$, this indicates that constraints are satisfied. Each supplementary controller operates by forcing the global impedance response at the given location as shown in Fig. 8 (diagonal elements) to approximate the local impedances of Fig. 4. Fig. 16 depicts the reshaped global impedances after supplementary control. As can be seen, the original peaks in the global responses have been pushed down 


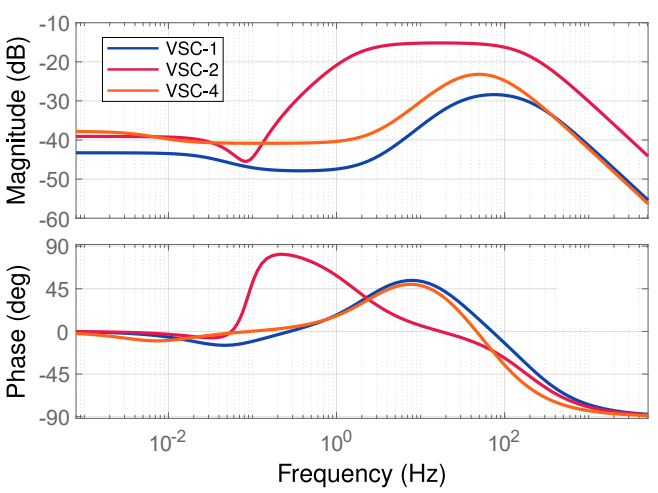

Fig. 15. Decentralized supplementary controllers at corresponding locations
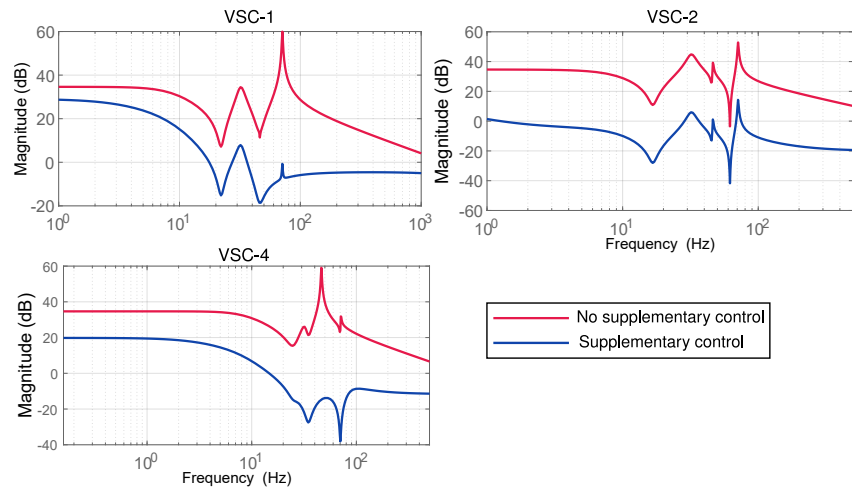

Fig. 16. Reshaped global impedances at each supplementary control terminal

significantly. Additionally, the synthesized controllers were able to closely approximate the local impedances at VSC-1 and VSC-4. The supplementary controller at VSC-1 peaks at $73 \mathrm{~Hz}$ around the same location as the dominant frequency identified in Fig. 9 for VSC-1. The supplementary control at VSC-4 peaks at $44 \mathrm{~Hz}$ similar to its dominant frequency identified in Fig. 9. Whereas at VSC-2 the approximation was poor due to the low order approximation of the target sensitivity function. An ideal controller at VSC-2 should have a low peak at the first interaction frequency and a higher peak at the second interaction frequency. However, this will result in a higher-order controller at VSC-2 which is unnecessary. Notwithstanding, this is not an issue as the magnitude of oscillatory frequencies has been significantly reduced and the dominant frequency at VSC-2 is dealt with by VSC-1.

To demonstrate the efficacy of the implemented controllers in decoupling interactions, Fig. 17 shows the new RGA profile of the network for all three designed controllers. It can be seen in the dynamic interaction region, that all diagonal elements approach 1 and correspondingly, off-diagonal elements approach 0 (unit matrix). This indicates a complete decoupling of the interactions in the indicated region. This is despite only three controllers in a four-terminal network. It will be shown in the following that only two controllers are sufficient depending on the operating point.
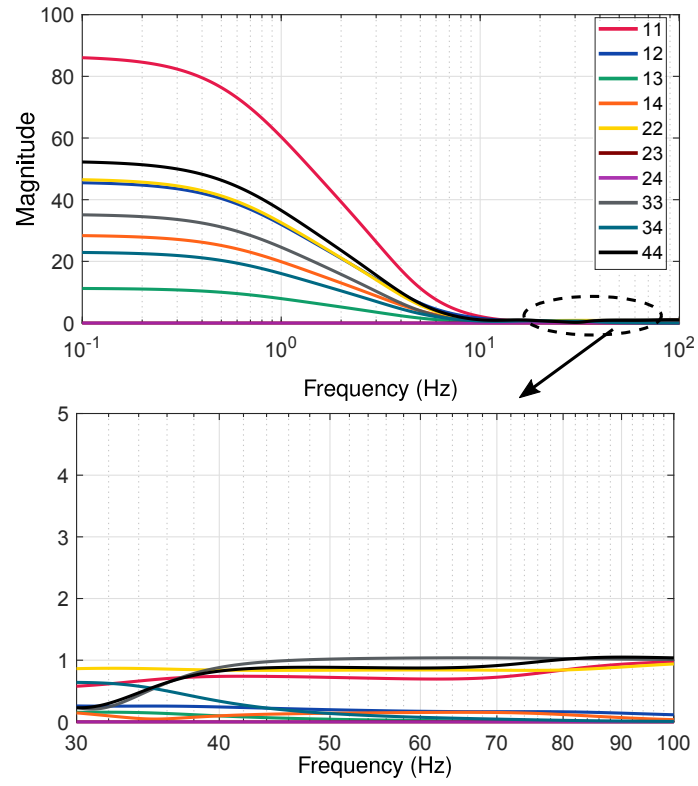

Fig. 17. Modified frequency-dependent relative gain array profile of the system with all three controllers activated
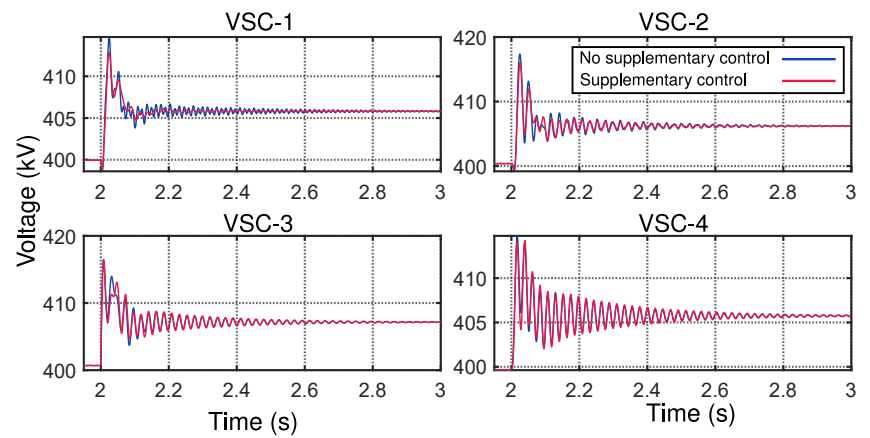

Fig. 18. Network DC voltage response for controller located at VSC-1 for simultaneous step disturbances at the offshore resource and VSC-1

\section{A. Impact of one Supplementary Controller}

For a single supplementary controller in the system located at VSC-1 with the rest deactivated, Fig. 18 shows the grid DC voltage responses for simultaneous step disturbances from terminals VSC-1 and the offshore resource at VSC-3. To obtain insights into responses shown, recall that for disturbances from VSC-1 and VSC-3 the dominant interaction frequencies in the system are at $73 \mathrm{~Hz}$ and $44.2 \mathrm{~Hz}$ respectively. However, since the controller at VSC-1 was designed considering the dominant mode as seen from VSC-1 only the $73 \mathrm{~Hz}$ component will be damped leaving the $44.2 \mathrm{~Hz}$ component untouched. This can be seen from the time-domain simulation in Fig. 18 where there is only an improvement in responses is at VSC-1 whereas, at terminals VSC-3 and VSC-4 there is no improvement as the dominant mode is at $44.2 \mathrm{~Hz}$.

For another case of a single supplementary controller located instead at VSC-4 Fig. 19 shows the responses for disturbance from the offshore resource showing a significant improvement. This is due to the supplementary controller designed to mitigate interactions from its vicinity and that from the offshore resource in VSC-3. As for VSC-1 only the 44.2 


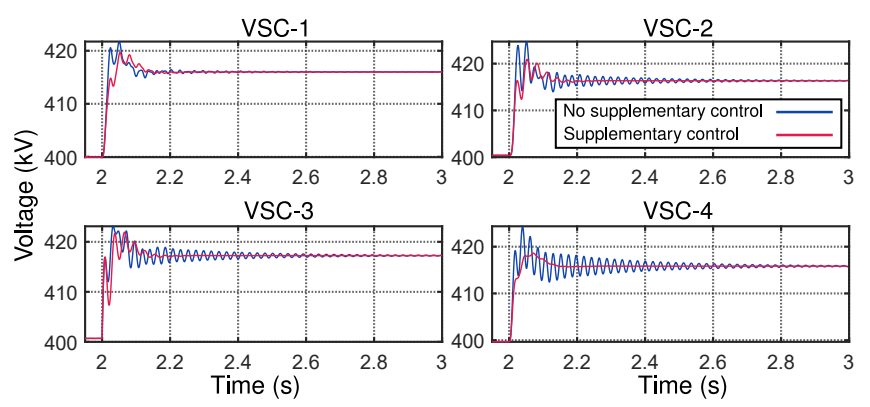

Fig. 19. Network DC voltage responses for controller located at VSC-4 for step disturbances at the offshore resource
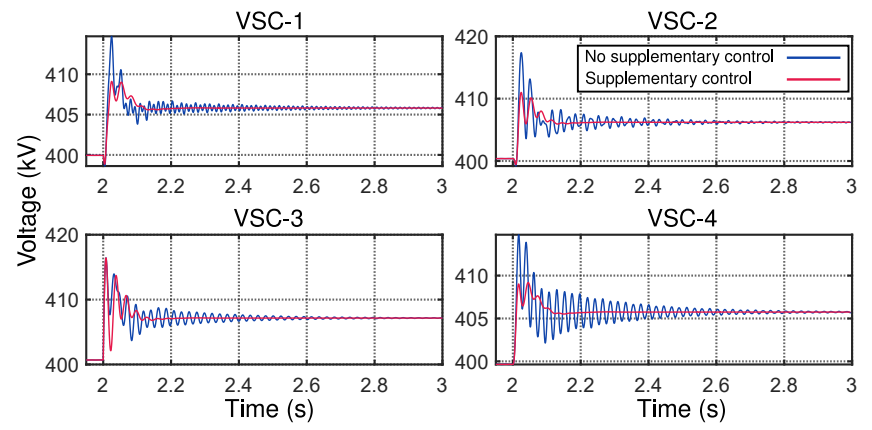

Fig. 20. Network DC voltage responses for two controllers located at VSC-1 and VSC-4 and simultaneous disturbances at the offshore resource and VSC-1

$\mathrm{Hz}$ component is eliminated; hence only partial improvements. Therefore, to cover all potential sources of disturbances in this system, at least two supplementary controllers are required particularly at terminals VSC- 1 and VSC- 4 or VSC- $1 / 2$ and VSC-4.

\section{B. Impact of two Supplementary Controllers}

To confirm the hypothesis made in the previous subsection, two supplementary controllers at VSC- 1 and VSC-4 are activated to cover all sources of disturbances. Fig. 20 shows the DC voltage responses for disturbances from VSC-1 and VSC3. The improvements over the case without supplementary controllers are clear as the dominant interaction modes in the system have been damped out. Additionally, despite the lack of a supplementary controller at VSC-2 and the offshore resource in VSC-3, the response is much improved compared to without the supplementary controller and that of Fig. 18. This shows the influence of only two supplementary controllers in covering the entire range of potential interaction frequencies. Hence, any additional supplementary controller will simply be redundant with negligible effects. This is confirmed in Fig. 21 where there is no significant improvement of the three supplementary controllers compared to two.

\section{Performance of Controllers Under Single-line/Converter Disconnection}

To demonstrate the robustness and dynamic performance of the synthesized controllers to unexpected changes, Fig. 22 shows the comparison of DC voltage responses for disconnection of the line connecting VSC-1 and VSC- 2 at $2 \mathrm{~s}$. This

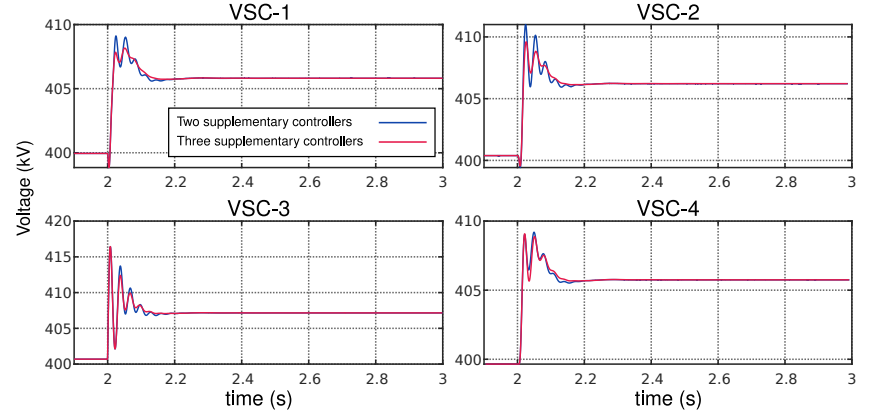

Fig. 21. Network DC voltage responses for two controllers located at VSC-1 and VSC-4 and all three synthesized controllers for simultaneous disturbances at the offshore resource and VSC-1
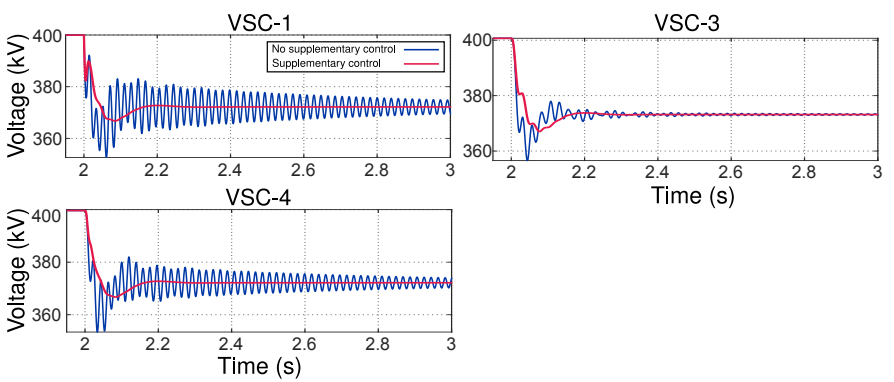

Fig. 22. Network DC voltage responses for disconnection of line between VSC-1 and VSC-2 with two supplementary controllers at VSC-1 and VSC-4
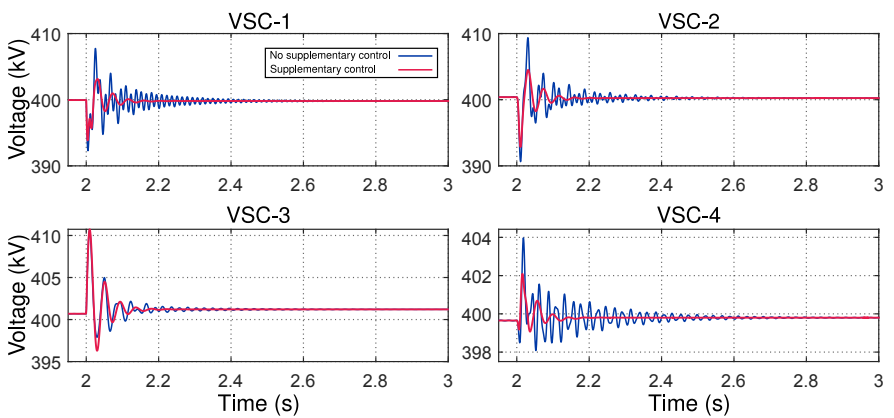

Fig. 23. Network DC voltage responses for disconnection of line between VSC-1 and VSC-3 with two supplementary controllers at VSC-1 and VSC-4

also disconnects VSC-2 from the HVDC grid. For this case, two supplementary controllers at VSC-1 and VSC-4 (with the same parameters as all previous cases) are active. The impact of supplementary control is obvious without direct adaptation of parameters. The supplementary controllers facilitated the ease of transition to the new operating point while damping interaction induced oscillations. To remark, in a real physical grid, the parameters of the controllers would need to be adapted on-line after a certain period post-disturbance (for disturbances that change the topology). This is to account for the new state of the grid, ahead of the next potential (unknown) event. In a less severe case, Fig. 23 shows a similar comparison for the disconnection of the line connecting the offshore resource at VSC-3 and VSC-1 under the same conditions as previously. It can be seen that despite only two controllers being activated, the dynamic responses are significantly better and less distorted with the supplementary controllers. 


\section{CONCLUSION}

The methodology presented in this paper provides a systematic methodology for system-wide interaction analysis and control design in VSC-HVDC systems with different converters. The converter models may be based on black-box transfer functions; therefore, critical information is not required. Additionally, the interaction analysis presented detects precisely how each terminal in the grid is interacting with others relative to the magnitudes. This directly keys into the coordination of designed supplementary controllers and the simplicity of design. Simulation results on a four-terminal VSC-HVDC grid demonstrate the flexibility and efficacy of the proposed methodology for decentralized low order supplementary controllers. However, a potential drawback of the control design procedures based on impedance matching is the choice of output weighting functions for large and highorder systems. In many cases, this may require an efficient method to obtain effective low-order weights, since such is a prerequisite for low order controller synthesis. Moreover, there is an added cost of implementing additional controllers at each controllable node. In summary, the methodology can be applied in detection, analysis, and mitigation of network-wide interactions as a result of the independent design of converter controllers.

TABLE III

GRID PARAMETERS

\begin{tabular}{cc}
\hline Parameter & Value \\
\hline Rated power & $800 \mathrm{MW}$ \\
Rated AC-side voltage & $400 \mathrm{kV}$ \\
Rated AC-side voltage & $220 \mathrm{kV}(\mathrm{rms} \mathrm{L}-\mathrm{L})$ \\
Grid short-circuit ratio & 9 \\
Filter impedance & $0.004+j 0.1$ p.u. \\
Transformer impedance & $j 0.12$ p.u. \\
Equivalent DC capacitance & $120 \mu \mathrm{F}$ \\
\hline
\end{tabular}

TABLE IV

CONTROL DATA

\begin{tabular}{ccc}
\hline Controller & Time constant & Damping ratio \\
\hline AC-side Current & $1 \mathrm{~ms}$ & 0.7071 \\
AC-side voltage & $100 \mathrm{~ms}$ & 0.7071 \\
Phase-locked loop (PLL) & $20 \mathrm{~ms}$ & 0.7071 \\
\hline
\end{tabular}

\section{REFERENCES}

[1] D. V. Hertem and M. Ghandhari, "Multi-terminal vsc hvdc for the european supergrid: Obstacles," Renewable and Sustainable Energy Reviews, vol. 14, no. 9, pp. $3156-3163,2010$.

[2] P. Rault, "Implementation of a dedicated control to limit adverse interaction in multi-vendor hvdc systems," IET Conference Proceedings, pp. 7 (6 pp.)-7 (6 pp.)(1), January 2019

[3] B. Tourgoutian and A. Alefragkis, "Design considerations for the cobracable hvdc interconnector," in IET International Conference on Resilience of Transmission and Distribution Networks (RTDN 2017), Sep. 2017, pp. 1-7.

[4] V. Staudt, A. Steimel, M. Kohlmann, M. K. Jger, C. Heising, D. Meyer, K. Vennemann, E. Grebe, and K. Kleinekorte, "Control concept including validation strategy for an ac/dc hybrid link (ultranet)," in 2014 IEEE Energy Conversion Congress and Exposition (ECCE), Sep. 2014, pp. 750-757.
[5] B. P. Project. (2018) Final recommendations for interoperability of multivendor hvdc systems. [Online]. Available: http://www.bestpaths-project.eu/contents/publications/ d93--final-demo2-recommendations--vfinal.pdf

[6] X. Li, Z. Yuan, J. Fu, Y. Wang, T. Liu, and Z. Zhu, "Nanao multiterminal vsc-hvdc project for integrating large-scale wind generation," in 2014 IEEE PES General Meeting - Conference Exposition, July 2014, pp. $1-5$.

[7] S. D'Arco, J. A. Suul, and M. Molinas, "Implementation and analysis of a control scheme for damping of oscillations in vsc-based hvdc grids," in Power Electronics and Motion Control Conference and Exposition (PEMC), 2014 16th International, Sept 2014, pp. 586-593.

[8] J. Beerten, S. D'Arco, and J. A. Suul, "Cable model order reduction for hvdc systems interoperability analysis," in $A C$ and $D C$ Power Transmission, 11th IET International Conference on, Feb 2015, pp. 110.

[9] J. Beerten, S. D'Arco, and J. A. Suul, "Identification and small-signal analysis of interaction modes in vsc mtdc systems," IEEE Transactions on Power Delivery, vol. 31, no. 2, pp. 888-897, April 2016.

[10] M. Amin, A. Rygg, and M. Molinas, "Impedance-based and eigenvalue based stability assessment compared in vsc-hvdc system," in 2016 IEEE Energy Conversion Congress and Exposition (ECCE), Sept 2016, pp. 18.

[11] K. Sharifabadi, L. Harnefors, H.-P. Nee, S. Norrga, and R. Teodorescu, Design, control, and application of modular multilevel converters for HVDC transmission systems. John Wiley \& Sons, 2016.

[12] A. J. Agbemuko, J. L. Domínguez-García, and O. Gomis-Bellmunt, "An integrated approach to understanding the impact of network resonances and control on dynamic responses in vsc-hvdc networks," IFACPapersOnLine, vol. 51, no. 28, pp. 344 - 349, 2018.

[13] A. J. Agbemuko, J. L. Domínguez-García, E. Prieto-Araujo, and O. Gomis-Bellmunt, "Dynamic modelling and interaction analysis of multi-terminal vsc-hvdc grids through an impedance-based approach," International Journal of Electrical Power \& Energy Systems, vol. 113, pp. $874-887,2019$.

[14] H. Saad, J. Mahseredjian, S. Dennetire, and S. Nguefeu, "Interactions studies of HVDCMMC link embedded in an AC grid," Electric Power Systems Research, vol. 138, pp. 202 - 209, 2016.

[15] H. Saad, Y. Fillion, S. Deschanvres, Y. Vernay, and S. Dennetire, "On resonances and harmonics in HVDC-MMC station connected to AC grid," IEEE Transactions on Power Delivery, vol. 32, no. 3, pp. 15651573, June 2017.

[16] E. Prieto-Araujo, A. Egea-Alvarez, S. Fekriasl, and O. Gomis-Bellmunt, "DC voltage droop control design for multiterminal HVDC systems considering AC and DC grid dynamics," IEEE Transactions on Power Delivery, vol. 31, no. 2, pp. 575-585, April 2016.

[17] J. Freytes, Small-signal stability analysis of Modular Multilevel Converters and application to MMC-based Multi-Terminal DC grids, 2017.

[18] J. J. Grainger and W. D. Stevenson Jr., Power system analysis. McGrawHill International Editions, 1994.

[19] S. Skogestad and I. Postlethwaite, Multivariable feedback control: analysis and design. Wiley New York, 2007, vol. 2.

[20] E. Bristol, "On a new measure of interaction for multivariable process control," IEEE Transactions on Automatic Control, vol. 11, no. 1, pp. 133-134, January 1966.

[21] M. Hovd and S. Skogestad, "Simple frequency-dependent tools for control system analysis, structure selection and design," Automatica, vol. 28 , no. 5, pp. $989-996,1992$.

[22] P. Gahinet and P. Apkarian, "Decentralized and fixed-structure $\mathcal{H}_{\infty}$ control in matlab," in 2011 50th IEEE Conference on Decision and Control and European Control Conference, Dec 2011, pp. 8205-8210. 


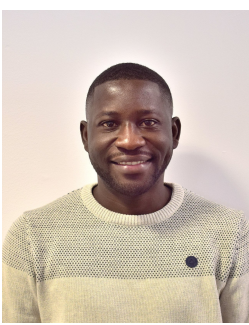

Adedotun J. Agbemuko (S'16-M'20) received the BEng. degree in electrical engineering from the Federal University of Technology, Minna (FUT Minna), Nigeria, in 2012, the MSc. degree in electrical engineering from the Delft University of Technology, Delft (TU Delft), the Netherlands, in 2016, and the Ph.D. degree (cum laude) in electrical engineering from Technical University of Catalonia (UPC), Barcelona, Spain in 2019. Since 2016, he has been with the power systems research group at Institut de Recerca en Energia de Catalunya (IREC). His research interests include modelling, network-scale interaction analysis and control of grid-connected converters, network modelling and control of largescale hybrid AC/DC grids including multi-terminal HVDC grids.

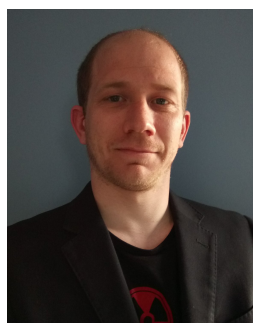

Jose Luis Domínguez-García received the B.Sc. and M.Sc. degrees in industrial engineering from the School of Industrial Engineering of Barcelona, Universitat Politécnica de Catalunya (UPC), Barcelona, Spain, in 2009, the masters thesis in Oulun Yliopisto, Oulu, Finland, and the Ph.D. degree (cum laude) in electrical engineering from UPC, in 2013. He was a Researcher Visitor with the Institute of Energy, Cardiff University, and the University of Strathclyde, U.K., in 2011 and 2016, respectively. In 2010, he joined IREC, Barcelona, Spain, as a Researcher, and is currently the Head of Power Systems Group. He has been awarded the Distinguished Visiting Fellowship from the Royal Academy of Engineering. He has authored more than 25 journal papers and coauthored 1 patent. He actively participates and coordinates several industrial and research projects focused on wind power integration, power system stability, and offshore grids design and control. His research interests include grid integration, control and modelling of renewable energies, transmission and distribution grid management, power converters, and power system dynamics. He was the recipient of the Outstanding Ph.D. Thesis award from UPC in 2015.

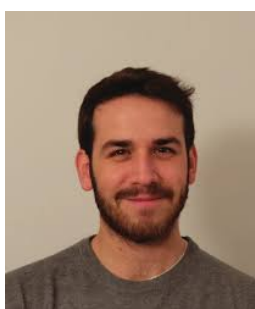

Eduardo Prieto-Araujo (S'12-M'16) received the degree in industrial engineering from the School of Industrial Engineering of Barcelona (ETSEIB), Technical University of Catalonia (UPC), Barcelona, Spain, in 2011 and the Ph.D. degree in electrical engineering from the UPC in 2016. He joined CITCEA-UPC research group in 2010 and currently he is a Serra Húnter Lecturer with the Electrical Engineering Department, UPC. His main interests are renewable generation systems, control of power converters for HVDC applications, interaction analysis between converters, and power electronics dominated power systems.

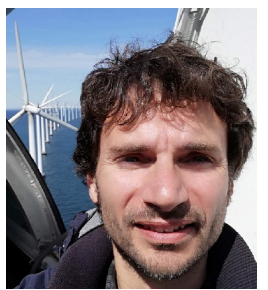

Oriol Gomis-Bellmunt (S'05-M'07-SM'12) received the degree in industrial engineering from the School of Industrial Engineering of Barcelona (ETSEIB), Technical University of Catalonia (UPC), Barcelona, Spain, in 2001 and the Ph.D. degree in electrical engineering from the UPC in 2007. In 1999, he joined Engitrol S.L. where he worked as Project Engineer in the automation and control industry. Since 2004, he has been with the Electrical Engineering Department, UPC where he is a Professor and participates in the CITCEA-UPC Research Group. Since 2020, he is an ICREA Academia researcher. His research interests include the fields linked with electrical machines, power electronics, and renewable energy integration in power systems. 